Document downloaded from:

http://hdl.handle.net/10251/34930

This paper must be cited as:

Sastre, J.; Ibáñez González, JJ.; Defez Candel, E.; Ruíz Martínez, PA. (2011). Efficient orthogonal matrix polynomial based method for computing matrix exponential. Applied Mathematics and Computation. 217(14):6451-6463. doi:10.1016/j.amc.2011.01.004.

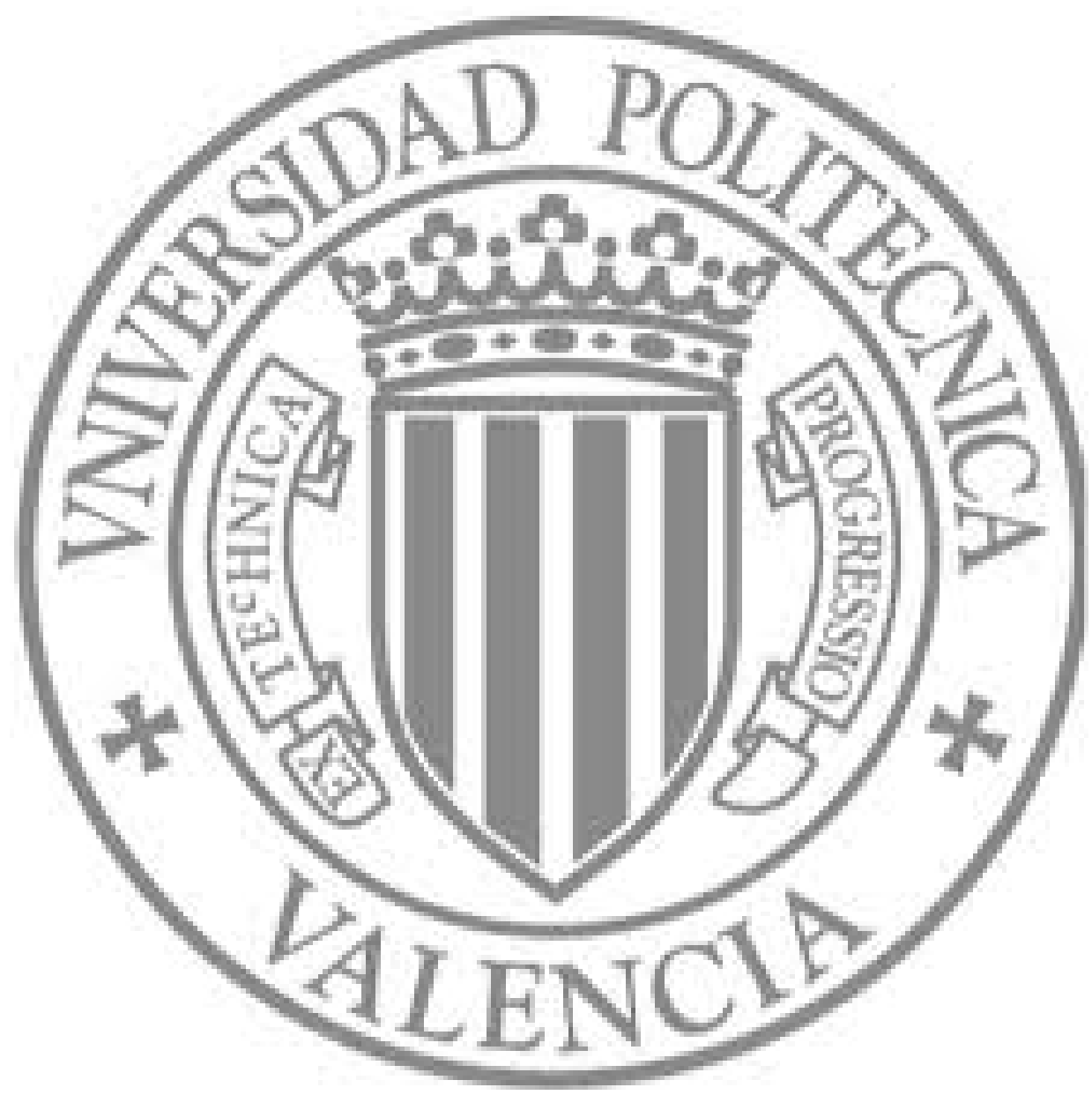

The final publication is available at

http://dx.doi.org/10.1016/j.amc.2011.01.004

Copyright Elsevier 


\title{
Efficient orthogonal matrix polynomial based method for computing matrix exponential
}

\author{
J. Sastre ${ }^{\mathrm{a}, *}$, J. Ibáñez ${ }^{\mathrm{b}}$, E. Defez ${ }^{\mathrm{c}}$, P. Ruiz ${ }^{\mathrm{b}}$ \\ ${ }^{a}$ Instituto de Telecomunicaciones y Aplicaciones Multimedia, \\ ${ }^{b}$ Instituto de Instrumentación para Imagen Molecular, \\ ${ }^{c}$ Instituto de Matemática Multidisciplinar, \\ Universitat Politècnica de València, Camino de Vera s/n, 46022-Valencia (Spain)
}

\begin{abstract}
The matrix exponential plays a fundamental role in the solution of differential systems which appear in different science fields. This paper presents an efficient method for computing matrix exponentials based on Hermite matrix polynomial expansions. Hermite series truncation together with scaling and squaring and the application of floating point arithmetic bounds to the intermediate results provide excellent accuracy results compared with the best acknowledged computational methods. A backward-error analysis of the approximation in exact arithmetic is given. This analysis is used to provide a theoretical estimate for the optimal scaling of matrices. Two algorithms based on this method have been implemented as MATLAB functions. They have been compared with MATLAB functions funm and expm obtaining greater accuracy in the majority of tests. A careful cost comparison analysis with expm is provided showing that the proposed algorithms have lower maximum cost for some matrix norm intervals. Numerical tests show that the application of floating point arithmetic bounds to the intermediate results may reduce considerably computational costs, reaching in numerical tests relative higher average costs than expm of only $4.43 \%$ for the final Hermite selected order, and obtaining better accuracy results in the $77.36 \%$ of the test matrices. The MATLAB implementation of the best Hermite matrix polynomial based algorithm has been made available online.
\end{abstract}

Keywords: differential equations, matrix exponential, Hermite matrix

\footnotetext{
${ }^{*}$ Corresponding author

Email address: jorsasma@iteam.upv.es (J. Sastre)
} 
polynomial approximation, matrix polynomial evaluation, error analysis PACS: 87.64.Aa

\section{Introduction}

Many engineering processes are described by systems of linear first-order ordinary differential equations. Solving this kind of systems involves the evaluation of the exponential of square matrices, and the same occurs with the partial differential case when using the semi-discretization method [1]-[5].

A survey of methods for computing the exponential matrix was made by Moler and Van Loan in [4], which was updated in [6]. This paper and recent researches $[7,8]$ show that probably one of the more promising methods is the scaling and squaring technique, which is also the most widely used. This technique exploits the relation $\exp (A)=\left(\exp \left(2^{-s} A\right)\right)^{2^{s}}$, for a square matrix $A$ and a nonnegative integer scaling parameter $s$. Most approximations to the exponential of the scaled matrix $\exp \left(2^{-s} A\right)$ are based on Padé expansions. [7] provides a scaling and squaring Padé method with excellent results of efficiency and accuracy, which is the method implemented in the last MATLAB versions, and [8] has recently proposed a new scaling and squaring algorithm that alleviates the overscaling problem for nonnormal matrices.

In this paper we use Hermite matrix polynomial expansions of the matrix exponential together with scaling and squaring and the application of floating point arithmetic bounds to the intermediate results in order to perform a competitive method for computing the matrix exponential. This method has the advantage that it does not need the solution of multiple linear systems or the computation of matrix inversions. Moreover, adapting the analysis made in [7] to the Hermite matrix series, a backward-error bound in exact arithmetic has been provided to find the optimal matrix scaling, i.e. the optimal value of scaling parameter $s$. A careful theoretical cost comparison with the method in [7] has been provided, showing that Hermite method has lower cost for some matrix norm intervals.

This paper is organized as follows. Section 2 summarizes notation and previous results about Hermite matrix polynomials and includes the Hermite series expansion of the matrix exponential to be considered. Section 3 deals with the approximation error analysis and optimal scaling. Section 4 is addressed to study the cost of the method, and to present numerical tests in order to check the method accuracy performance. Finally conclusions are given in Section 5. 


\section{Hermite matrix polynomial series expansions of matrix expo- nential}

Throughout this paper, for a complex number $z, \Re(z)$ and $\Im(z)$ denote its real and imaginary parts, respectively, and $\|\cdot\|$ denotes any subordinate matrix norm. $\mathbb{R}^{r \times r}$ and $\mathbb{C}^{r \times r}$ denote the set of real and complex matrices of size $r \times r$, respectively, and $I$ denotes the identity matrix for these sets. For a matrix $A \in \mathbb{C}^{r \times r}$, its spectrum $\sigma(A)$ denotes the set of all the eigenvalues of $A$. If $f(z)$ and $g(z)$ are holomorphic functions of the complex variable $z$, which are defined in an open set $\Omega$ of the complex plane, and $B$ is a matrix in $\mathbb{C}^{r \times r}$ with $\sigma(B) \subset \Omega$, then from the properties of the matrix functional calculus [9, p. 558], it follows that $f(B) g(B)=g(B) f(B)$. If $\mathbb{D}_{0}$ is the complex plane cut along the negative real axis and $\log (z)$ denotes the principal logarithm of $z$, [10, p. 72], then $z^{\frac{1}{2}}$ represents $\exp \left(\frac{1}{2} \log (z)\right)$. If $B$ is a matrix with $\sigma(B) \subset \mathbb{D}_{0}$, then $B^{\frac{1}{2}}=\sqrt{B}$ denotes the image by $z^{\frac{1}{2}}$ of the matrix functional calculus acting on the matrix $B$. We say that matrix $A$ in $\mathbb{C}^{r \times r}$ is a positive stable matrix if $\operatorname{Re}(z)>0$ for all $z \in \sigma(A)$. $[x]$ denotes the entire part of $x,\lceil x\rceil$ denotes the least integer not less than $x$ and $\lfloor x\rfloor$ denotes the greatest integer not exceeding $x$.

For the sake of clarity in the presentation of the next results we recall some properties and results about Hermite matrix polynomials that have been established in [11] and [12]. If $B$ is a positive stable matrix in $\mathbb{C}^{r \times r}$, the $n$th Hermite matrix polynomial is defined in $(3.4)$ of $[12$, p. 25] by

$$
H_{n}(x, B)=n ! \sum_{k=0}^{\left[\frac{n}{2}\right]} \frac{(-1)^{k}(x \sqrt{2 B})^{n-2 k}}{k !(n-2 k) !},
$$

and from (3.1) and (3.2) of [12, p. 24] one gets its generating function

$$
G(x, t)=e^{x t \sqrt{2 B}-t^{2} I}=\sum_{n \geq 0} H_{n}(x, B) t^{n} / n !, \quad|t|<\infty,
$$

where $x \in \mathbb{C}$ and $t \in \mathbb{C}$. Taking $A=\sqrt{2 B}, y=t x$ and $\lambda=1 / t$ in (2) it follows that

$$
e^{A y}=e^{\frac{1}{\lambda^{2}}} \sum_{n \geq 0} \frac{1}{\lambda^{n} n !} H_{n}\left(\lambda y, \frac{1}{2} A^{2}\right), \lambda \in \mathbb{C}, \quad y \in \mathbb{C}, \quad A \in \mathbb{C}^{r \times r},
$$


without restrictions on $\sigma(A)$. From (1) one gets

$$
H_{n}\left(\lambda y, A^{2} / 2\right)=n ! \sum_{k=0}^{\left[\frac{n}{2}\right]} \frac{(-1)^{k}(\lambda y A)^{n-2 k}}{k !(n-2 k) !},
$$

also without restrictions on $\sigma(A)$. Denoting by $h_{m}(\lambda y, A)$ the $m$-th partial sum of series (3), one gets

$$
h_{m}(\lambda y, A)=e^{\frac{1}{\lambda^{2}}} \sum_{n=0}^{m} \frac{1}{\lambda^{n} n !} H_{n}\left(\lambda y, \frac{1}{2} A^{2}\right) \approx e^{A y}, \quad \lambda, y \in \mathbb{C}, \quad A \in \mathbb{C}^{r \times r} .
$$

This expansion is the one to be used in the method for computing the matrix exponential, and we will refer to $m$ as the order of the approximation. Using (4) and (5) by induction it is easy to show that

$$
h_{m}(\lambda y, A)=\left\{\begin{array}{l}
e^{\frac{1}{\lambda^{2}}}\left[E_{0}^{\frac{m-1}{2}}(I+A y)+E_{0}^{\frac{m-1}{2}-1}\left(\frac{(A y)^{2}}{2 !}+\frac{(A y)^{3}}{3 !}\right)+\cdots\right. \\
\left.\cdots+E_{0}^{1}\left(\frac{(A y)^{m-3}}{(m-3) !}+\frac{(A y)^{m-2}}{(m-2) !}\right)+\frac{(A y)^{m-1}}{(m-1) !}+\frac{(A y)^{m}}{m !}\right], \text { odd } m \\
e^{\frac{1}{\lambda^{2}}}\left[E_{0}^{\frac{m}{2}} I+E_{0}^{\frac{m}{2}-1}\left(A y+\frac{(A y)^{2}}{2 !}\right)+E_{0}^{\frac{m}{2}-2}\left(\frac{(A y)^{3}}{3 !}+\frac{(A y)^{4}}{4 !}\right)+\cdots\right. \\
\left.\cdots+E_{0}^{1}\left(\frac{(A y)^{m-3}}{(m-3) !}+\frac{(A y)^{m-2}}{(m-2) !}\right)+\frac{(A y)^{m-1}}{(m-1) !}+\frac{(A y)^{m}}{m !}\right], \text { even } m
\end{array}\right.
$$

where

$$
E_{i}^{j}=\sum_{k=i}^{j} \frac{\left(-\frac{1}{\lambda^{2}}\right)^{k}}{k !}, i, j \in \mathbb{N}, j \geq i
$$

Note that for $|\lambda| \rightarrow \infty, e^{\frac{1}{\lambda^{2}}}=1, E_{0}^{j}=1$, and $h_{m}(\lambda y, A)$ tends to the Taylor series of order $m$ of matrix exponential $e^{A y}$.

\section{Error analysis.}

Using (6) with $y=1$ and taking into account Taylor series of $\exp \left(-\frac{1}{\lambda^{2}}\right)$, for odd $m$ it follows that

$$
\begin{aligned}
e^{A}-h_{m}(\lambda, A)= & \lim _{M \rightarrow \infty}\left(h_{M}(\lambda, A)-h_{m}(\lambda, A)\right) \\
= & e^{\frac{1}{\lambda^{2}}}\left[\sum_{k \geq \frac{m+1}{2}} \frac{\left(-\frac{1}{\lambda^{2}}\right)^{k}}{k !}(I+A)+\sum_{k \geq \frac{m-1}{2}} \frac{\left(-\frac{1}{\lambda^{2}}\right)^{k}}{k !}\left(\frac{A^{2}}{2 !}+\frac{A^{3}}{3 !}\right)+\cdots\right. \\
& \left.\cdots+\sum_{k \geq 1} \frac{\left(-\frac{1}{\lambda^{2}}\right)^{k}}{k !}\left(\frac{A^{m-1}}{(m-1) !}+\frac{A^{m}}{m !}\right)+\sum_{k \geq 0} \frac{\left(-\frac{1}{\lambda^{2}}\right)^{k}}{k !} \sum_{j \geq m+1} \frac{A^{j}}{j !}\right]
\end{aligned}
$$




$$
\begin{aligned}
= & e^{\frac{1}{\lambda^{2}}}\left[\sum_{k \geq \frac{m+1}{2}} \frac{\left(-\frac{1}{\lambda^{2}}\right)^{k}}{k !} e^{A}+E_{\frac{m-1}{2}}^{\frac{m-1}{2}}\left(\frac{A^{2}}{2 !}+\frac{A^{3}}{3 !}\right)+\right. \\
& \left.+E_{\frac{m-3}{2}}^{\frac{m-1}{2}}\left(\frac{A^{4}}{4 !}+\frac{A^{5}}{5 !}\right)+\cdots+E_{1}^{\frac{m-1}{2}}\left(\frac{A^{m-1}}{(m-1) !}+\frac{A^{m}}{m !}\right)+E_{0}^{\frac{m-1}{2}} \sum_{j \geq m+1} \frac{A^{j}}{j !}\right] \\
= & \left(1-e^{\frac{1}{\lambda^{2}}} E_{0}^{\frac{m-1}{2}}\right) e^{A}+e^{\frac{1}{\lambda^{2}}}\left[E_{\frac{m-1}{2}}^{\frac{m-1}{2}}\left(\frac{A^{2}}{2 !}+\frac{A^{3}}{3 !}\right)+E_{\frac{m-3}{2}}^{\frac{m-1}{2}}\left(\frac{A^{4}}{4 !}+\frac{A^{5}}{5 !}\right)+\cdots\right. \\
& \left.\cdots+E_{1}^{\frac{m-1}{2}}\left(\frac{A^{m-1}}{(m-1) !}+\frac{A^{m}}{m !}\right)+E_{0}^{\frac{m-1}{2}} \sum_{j \geq m+1} \frac{A^{j}}{j !}\right] .
\end{aligned}
$$

Analogously, using (6), for even $m$ one gets

$$
\begin{aligned}
e^{A}-h_{m}(\lambda, A)= & \left(1-e^{\frac{1}{\lambda^{2}}} E_{0}^{\frac{m}{2}}\right) e^{A}+e^{\frac{1}{\lambda^{2}}}\left[E_{\frac{m}{2}}^{\frac{m}{2}}\left(A+\frac{A^{2}}{2 !}\right)+E_{\frac{m}{2}-1}^{\frac{m}{2}}\left(\frac{A^{3}}{3 !}+\frac{A^{4}}{4 !}\right)\right. \\
& \left.+\cdots+E_{1}^{\frac{m}{2}}\left(\frac{A^{m-1}}{(m-1) !}+\frac{A^{m}}{m !}\right)+E_{0}^{\frac{m}{2}} \sum_{j \geq m+1} \frac{A^{j}}{j !}\right] .
\end{aligned}
$$

In a similar way to the demonstration of Theorem 2.1 of $[7$, p. 1182] we have the following result:

Theorem 3.1. Assume that the matrix exponential Hermite approximation $h_{m}(\lambda, A)$ of (5) satisfies

$$
e^{-2^{-s} A} h_{m}\left(\lambda, 2^{-s} A\right)=I+G
$$

where $\|G\|<1$. Then there exists a matrix $E$ that commutes with $A$ such that

$$
\left[h_{m}\left(\lambda, 2^{-s} A\right)\right]^{2^{s}}=e^{A+E}
$$

and

$$
\frac{\|E\|}{\|A\|} \leq \frac{-\log (1-\|G\|)}{\left\|2^{-s} A\right\|}
$$

We seek to bound the norm of $G$ in (10) in terms of $\left\|2^{-s} A\right\|$. Define the function

$$
\rho(\lambda, A)=e^{-A} h_{m}(\lambda, A)-I,
$$

and note that using (8), (9) and (13) one gets

$$
\rho(\lambda, A)=-e^{-A}\left(e^{A}-h_{m}(\lambda, A)\right)
$$




$$
=\left\{\begin{array}{l}
\left(e^{\frac{1}{\lambda^{2}}} E_{0}^{\frac{m-1}{2}}-1\right) I-e^{-A} e^{\frac{1}{\lambda^{2}}}\left[E_{\frac{m-1}{2}}^{\frac{m-1}{2}}\left(\frac{A^{2}}{2 !}+\frac{A^{3}}{3 !}\right)+E_{\frac{m-3}{2}}^{\frac{m-1}{2}}\left(\frac{A^{4}}{4 !}+\frac{A^{5}}{5 !}\right)+\right. \\
\left.+\cdots+E_{1}^{\frac{m-1}{2}}\left(\frac{A^{m-1}}{(m-1) !}+\frac{A^{m}}{m !}\right)+E_{0}^{\frac{m-1}{2}} \sum_{j \geq m+1} \frac{A^{j}}{j !}\right], \text { odd } m \\
\left(e^{\frac{1}{\lambda^{2}}} E_{0}^{\frac{m}{2}}-1\right) I-e^{-A} e^{\frac{1}{\lambda^{2}}}\left[E_{\frac{m}{2}}^{\frac{m}{2}}\left(A+\frac{A^{2}}{2 !}\right)+E_{\frac{m}{2}}^{\frac{m}{2}}\left(\frac{A^{3}}{3 !}+\frac{A^{4}}{4 !}\right)+\right. \\
\left.+\cdots+E_{1}^{\frac{m}{2}}\left(\frac{A^{m-1}}{(m-1) !}+\frac{A^{m}}{m !}\right)+E_{0}^{\frac{m}{2}} \sum_{j \geq m+1} \frac{A^{j}}{j !}\right], \text { even } m
\end{array}\right.
$$

Hence, taking into account (15), and using Taylor series of $e^{-A}$ one gets

$$
\left\|\rho\left(\lambda, 2^{-s} A\right)\right\| \leq\left\{\begin{array}{l}
\left|e^{\frac{1}{\lambda^{2}}} E_{0}^{\frac{m-1}{2}}-1\right|+e^{\Re\left(\frac{1}{\lambda^{2}}\right)} \sum_{j \geq 2}\left|c_{j}\right| \theta^{j}, \text { odd } m \\
\left|e^{\frac{1}{\lambda^{2}}} E_{0}^{\frac{m}{2}}-1\right|+e^{\Re\left(\frac{1}{\lambda^{2}}\right)} \sum_{j \geq 1}\left|c_{j}^{\prime}\right| \theta^{j}, \text { even } m
\end{array}\right.
$$

where $\theta=\left\|2^{-s} A\right\|$. Note that using (15) for $|\lambda| \rightarrow \infty$ it follows that

$$
\|\rho(\lambda, A)\|=\left\|-e^{-A} \sum_{j \geq m+1} \frac{A^{j}}{j !}\right\|=\left\|e^{-A} \sum_{j=0}^{m} \frac{A^{j}}{j !}-I\right\|,|\lambda| \rightarrow \infty,
$$

which is the corresponding bound for Taylor series approximation of matrix exponential. Using (10), (15) and (16) it follows that

$$
\|G\|=\left\|\rho\left(\lambda, 2^{-s} A\right)\right\| \leq f_{m}(\lambda, \theta),
$$

where $f_{m}(\lambda, \theta)$ is given by the expressions on the right hand side of (16) depending on $m$ being odd or even. Note that taking $|\lambda| \rightarrow \infty$, using (17), (18) and Taylor expansion of $e^{-A}$, it follows that

$$
\|G\|=\left\|\rho\left(\lambda, 2^{-s} A\right)\right\| \leq f_{m}(|\lambda| \rightarrow \infty, \theta)
$$

where

$$
f_{m}(|\lambda| \rightarrow \infty, \theta)=\sum_{j \geq m+1}\left|c_{j}^{\prime \prime}\right| \theta^{j}
$$

which is the corresponding bound for Taylor approximation of matrix exponential. Combining (12) with (18) one gets

$$
\frac{\|E\|}{\|A\|} \leq \frac{-\log \left(1-f_{m}(\lambda, \theta)\right)}{\theta} \text {. }
$$




\begin{tabular}{|c|cccccccc|}
\hline$m$ & 1 & 2 & 3 & 4 & 5 & 6 & 7 & 8 \\
$\theta_{m}^{\infty}$ & $2.220 \mathrm{e}-16$ & $2.581 \mathrm{e}-8$ & $1.386 \mathrm{e}-5$ & $3.397 \mathrm{e}-4$ & $2.401 \mathrm{e}-3$ & $9.066 \mathrm{e}-3$ & $2.384 \mathrm{e}-2$ & $4.991 \mathrm{e}-2$ \\
\hline \hline$m$ & 9 & 10 & 11 & 12 & 13 & 14 & 15 & 16 \\
$\theta_{m}^{\infty}$ & $8.958 \mathrm{e}-2$ & $1.448 \mathrm{e}-1$ & $2.142 \mathrm{e}-1$ & $2.996 \mathrm{e}-1$ & $3.998 \mathrm{e}-1$ & $5.139 \mathrm{e}-1$ & $6.411 \mathrm{e}-1$ & $7.803 \mathrm{e}-1$ \\
\hline \hline$m$ & 17 & 18 & 19 & 20 & 21 & 22 & 23 & 24 \\
$\theta_{m}^{\infty}$ & $9.305 \mathrm{e}-1$ & 1.091 & 1.260 & 1.438 & 1.624 & 1.816 & 2.015 & 2.219 \\
\hline \hline$m$ & 25 & 26 & 27 & 28 & 29 & 30 & & \\
$\theta_{m}^{\infty}$ & 2.429 & 2.643 & 2.861 & 3.084 & 3.310 & 3.540 & & \\
\hline
\end{tabular}

Table 1: Maximal values $\theta_{m}^{\infty}$ of $\left\|2^{-s} A\right\|$ such that the backward error bound (21) does not exceed $u=2^{-53}$ for $|\lambda| \rightarrow \infty$, i.e. Taylor expansion of matrix exponential.

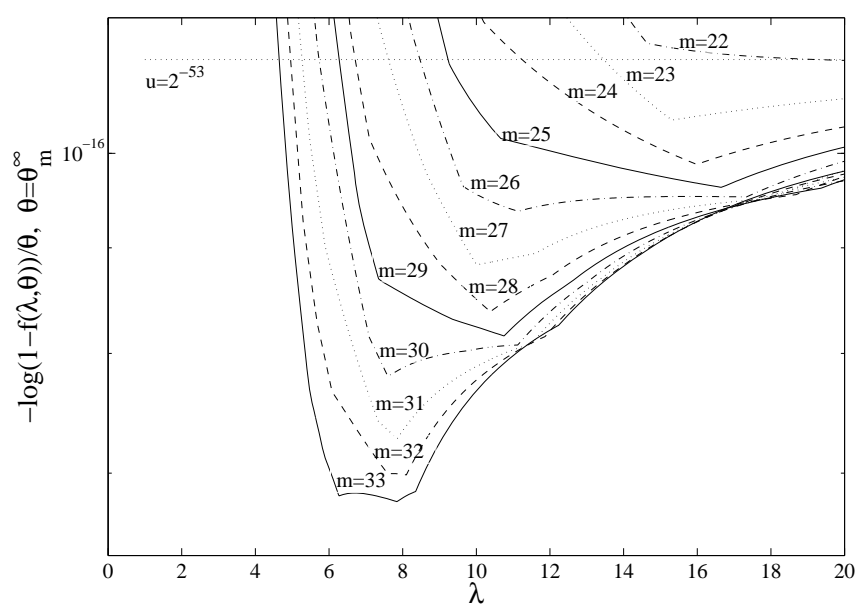

Figure 1: Bound (21) for $m=22,23, \ldots, 33$ and $\theta=\theta_{m}^{\infty}$, vs. parameter $\lambda$.

Using MATLAB's Symbolic Math Toolbox we have evaluated $f_{m}(\lambda, \theta)$ for (18) and (20), in 250 decimal digit arithmetic, summing the first 150 series terms in both expressions, where the coefficients $c_{j}, c_{j}^{\prime}$ and $c_{j}^{\prime \prime}$ have been obtained symbolically, $c_{j}, c_{j}^{\prime}$ being functions of parameter $\lambda$.

For $m=1,2, \ldots, 30$ we have used a zero-finder to determine the largest value of $\theta$, denoted by $\theta_{m}^{\infty}$, such that the backward error bound (21) for $|\lambda| \rightarrow \infty$ does not exceed the unit roundoff $u$ in IEEE double precision arithmetic, $u=2^{-53}$. These values correspond to Taylor approximation of matrix exponential. Table 1 presents the results with 4 significant digits.

Substituting these values in (21) and varying parameter $\lambda$, experimen- 


\begin{tabular}{|l|ccccccccc|}
\hline$m$ & 22 & 23 & 24 & 25 & 26 & 27 & 28 & 29 & 30 \\
\hline$\theta_{m}$ & 1.816 & 2.020 & 2.229 & 2.441 & 2.659 & 2.884 & 3.113 & 3.342 & 3.579 \\
\hline$\lambda$ & 26.904 & 15.311 & 15.986 & 16.661 & 11.074 & 10.009 & 10.381 & 10.753 & 7.596 \\
\hline
\end{tabular}

Table 2: Maximal values $\theta_{m}$ of $\left\|2^{-s} A\right\|$ such that the backward error bound (21) does not exceed $u=2^{-53}$ and values of $\lambda$ for which this is accomplished.

\begin{tabular}{|c|c|}
\hline$m$ & $h_{m}(\lambda, A)$ \\
\hline 4 & $\left(F_{0}^{0} / 4 ! A^{2}+F_{0}^{0} / 3 ! A+F_{0}^{1} / 2 I\right) A^{2}+F_{0}^{1} A+F_{0}^{2} I$ \\
\hline \multirow{2}{*}{6} & $\left(\left(F_{0}^{0} / 6 ! A^{2}+F_{0}^{0} / 5 ! A+F_{0}^{1} / 4 ! I\right) A^{2}+F_{0}^{1} / 3 ! A+F_{0}^{2} / 2 I\right) A^{2}+$ \\
& $F_{0}^{2} A+F_{0}^{3} I$ \\
\hline \multirow{2}{*}{9} & $\left(\left(F_{0}^{0} / 9 ! A^{3}+F_{0}^{0} / 8 ! A^{2}+F_{0}^{1} / 7 ! A+F_{0}^{1} / 6 ! I\right) A^{3}+F_{0}^{2} / 5 ! A^{2}+\right.$ \\
& $\left.F_{0}^{2} / 4 ! A+F_{0}^{3} / 3 ! I\right) A^{3}+F_{0}^{3} / 2 A^{2}+F_{0}^{4}(A+I)$ \\
\hline
\end{tabular}

Table 3: Evaluation of $h_{m}(\lambda, A)$, where $F_{0}^{n}=e^{\frac{1}{\lambda^{2}}} E_{0}^{n}$. Set $e^{\frac{1}{\lambda^{2}}}=1$ and $F_{0}^{n}=1$ to obtain the Taylor approximation.

tally the bound (21) is monotonically decreasing to $u$ for $\lambda>0$ and $m=$ $1,2, \ldots, 21$, and it has minima lower than $u$ for $6<\lambda<27$ and $m=$ $22,23, \ldots, 30$, see figure 1 . So it is possible to determine $\theta_{m}>\theta_{m}^{\infty}$ for $m \geq 22$ searching for those minima. We have used an iterative process and a minimum finder to obtain the new maximum values of $\theta_{m}, m=22,23, \ldots, 30$, and the minima of $\lambda>0$ for which the backward error bound (21) does not exceed $u$. Table 2 presents the results for the new $\theta_{m}$ values with 4 significant digits. Therefore, we will consider Taylor approximation of matrix exponential for $m \leq 21$ and Hermite approximation for $m \geq 22$.

From pages 73-74 and Table 4.1 of [14, p. 74], using Horner's and Paterson-Stockmeyer's methods [13], we can evaluate a matrix polynomial of degree $m$

$$
P_{m}(A)=\sum_{k=0}^{m} p_{k} A^{k}
$$

maximizing the degree of the polynomial for a given number of matrix product evaluations, for $m^{*}=\{1,2,4,6,9,12,16,20,25,30,36, \ldots\}$, i.e. for $m=k^{2}, k=1,2,3, \ldots$, and $m=k^{2}-k, k=2,3, \ldots$, using next evaluation 
formula

$$
\begin{aligned}
P_{m}(A)= & \left(\cdots\left(A^{q} p_{m}+A^{q-1} p_{m-1}+\cdots+A p_{m-q+1}+I p_{m-q}\right)\right. \\
\times & \left.A^{q}+A^{q-1} p_{m-q-1}+A^{q-2} p_{m-q-2}+\cdots+A p_{m-2 q+1}+I p_{m-2 q}\right) \\
& \cdots \cdots \\
\times & A^{q}+A^{q-1} p_{q-1}+A^{q-2} p_{q-2}+\cdots+A p_{1}+I p_{0},
\end{aligned}
$$

calculating and saving previously the matrix powers $A^{2}, A^{3}, \ldots, A^{q}$, where one can take $q=\lceil\sqrt{m}$ or $q=\lfloor\sqrt{m}\rfloor$. The even matrix powers can be calculated as $A^{2}=A A, A^{4}=A^{2} A^{2}, A^{6}=A^{4} A^{2}, \ldots$, and the odd matrix powers as $A^{2 k+1}=A A^{2 k}, k=1,2, \ldots$ Using (6), (7) and (23), Table 3 presents the evaluation formula of $h_{m}(\lambda, A)$ for some $m \in m^{*}$, where we use the coefficients $F_{0}^{n}=e^{\frac{1}{\lambda^{2}}} E_{0}^{n}$ and we selected $q=\lfloor\sqrt{m}\rfloor$ to minimize the number of matrix powers in memory. Note that one can obtain Taylor approximation from this table setting $e^{\frac{1}{\lambda^{2}}}=1$ and $F_{0}^{n}=1$. On the other hand, given parameter $\lambda$, the coefficients of the matrix powers in the approximations $h_{m}(\lambda, A)$ can be evaluated only once in 250 digit precision arithmetic before being rounded to IEEE double precision arithmetic. Evaluating $h_{m}(\lambda, A)$ for the rest of values of $m$ in a similar way, we determine the cost of evaluating $h_{m}(\lambda, A)$, in terms of matrix products. Selecting the optimal scaling parameter as $s=\left\lceil\log _{2}\|A\| / \theta_{m}\right\rceil$ if $\|A\| \geq \theta_{m}$, and $s=0$ otherwise, the cost of the algorithm in matrix multiplications is

$$
\pi_{m}+s=\pi_{m}+\max \left(\left\lceil\log _{2}\|A\|-\log _{2} \theta_{m}\right\rceil, 0\right),
$$

where $\pi_{m}$ denotes the number of matrix products evaluated to obtain Taylor or Hermite matrix polynomial approximations. It is important to note that the lower the $\theta_{m}$ values are, the larger the number of final squaring steps are necessary. Considering $\|A\| \geq \theta_{m}$ and ignoring the constant shift $\|A\|$ we have to minimize

$$
C_{m}=\pi_{m}-\log _{2} \theta_{m},
$$

in order to obtain the best choice for $m$ in the Hermite approximation, see [7, p. 1184]. Considering Taylor approximation of matrix exponential for $m<22$ and therefore $\theta_{m}=\theta_{m}^{\infty}, m<22$, table 4 presents the values $C_{m}$ and $\pi_{m}$ for $m=1,2, \ldots, 30$.

From table $4, C_{m}$ has an absolute minimum for $m=16$, and local minima for $m=9,12,20,25,30$, which correspond with optimal values in terms of number of matrix products. Thus, considering this analysis the optimal 


\begin{tabular}{|c|ccccccccccccccc|}
\hline$m$ & 1 & 2 & 3 & 4 & 5 & 6 & 7 & 8 & 9 & 10 & 11 & 12 & 13 & 14 & 15 \\
\hline$\pi_{m}$ & 0 & 1 & 2 & 2 & 3 & 3 & 4 & 4 & 4 & 5 & 5 & 5 & 6 & 6 & 6 \\
$C_{m}$ & 52.00 & 26.21 & 18.14 & 13.52 & 11.70 & 9.79 & 9.39 & 8.32 & 7.48 & 7.79 & 7.22 & 6.74 & 7.32 & 6.96 & 6.64 \\
\hline \hline$m$ & 16 & 17 & 18 & 19 & 20 & 21 & 22 & 23 & 24 & 25 & 26 & 27 & 28 & 29 & 30 \\
\hline$\pi_{m}$ & 6 & 7 & 7 & 7 & 7 & 8 & 8 & 8 & 8 & 8 & 9 & 9 & 9 & 9 & 9 \\
$C_{m}$ & 6.36 & 7.10 & 6.87 & 6.67 & 6.48 & 7.30 & 7.14 & 6.99 & 6.84 & 6.71 & 7.59 & 7.47 & 7.36 & 7.26 & 7.16 \\
\hline
\end{tabular}

Table 4: Number of matrix multiplications, $\pi_{m}$. Measure of overall $\operatorname{cost} C_{m}$.

\begin{tabular}{cccc|ccc}
\hline & Hermite & & & \multicolumn{3}{c}{ Padé } \\
\hline$m$ & $\theta_{m}$ & $\lambda$ & $\pi_{m}$ & $m$ & $\theta_{m}^{\prime}$ & $\pi_{m}^{\prime}$ \\
\hline 4 & $3.397168839976962 \mathrm{e}-4$ & - & 2 & 3 & $1.495585217958292 \mathrm{e}-2$ & 2 \\
6 & $9.065656407595101 \mathrm{e}-3$ & - & 3 & 5 & $2.539398330063230 \mathrm{e}-1$ & 3 \\
9 & $8.957760203223343 \mathrm{e}-2$ & - & 4 & 7 & $9.504178996162932 \mathrm{e}-1$ & 4 \\
12 & $2.996158913811581 \mathrm{e}-1$ & - & 5 & 9 & 2.097847961257068 & 5 \\
16 & $7.802874256626574 \mathrm{e}-1$ & - & 6 & 13 & 5.371920351148152 & 6 \\
20 & 1.438252596804337 & - & 7 & & & \\
25 & 2.441356829252848 & 16.66121324200387 & 8 & & & \\
30 & 3.578700513755017 & 7.596210771817034 & 9 & & & \\
\hline
\end{tabular}

Table 5: Theoretical optimal values of $\theta_{m}$ for Hermite method ( $m=$ order of approximation, $\pi_{m}=$ number of matrix products). For $m \leq 20, \theta_{m}=\theta_{m}^{\infty}$, i.e. the values relating to Taylor approximation. For $m=25,30, \theta_{m}$ have been obtained for the Hermite approximation, with the corresponding optimal values of $\lambda$. Parameter comparison with Padé expm method.

order, meaning with minimal cost, would be $m=16$. Table 5 presents the corresponding values of $\theta_{m}$ in IEEE double precision arithmetic and a comparison with the same values for Padé method proposed in [7]. From Table 5 one gets that $\theta_{20}<2 \theta_{16}$. Thus, if $m=20$ and the resulting scaling parameter is $s \geq 1$ then there exist matrices such that $\theta_{20} / 2<\left\|A / 2^{s}\right\| \leq$ $\theta_{16}$, and for such matrices one can use order $m=16$ instead of 20 in the approximation, saving one matrix product. The same occurs with orders $m=25$ and 20 , and $m=30$ and 25 and we will take this into account in the Hermite based algorithms. We will show in section 4 that with this modification the optimal maximum order is $m=20$ instead of $m=16$.

With respect to rounding errors, we rule out $m=1$ and 2 as maximum orders, as Taylor approximation can suffer from loss of significance in floating point arithmetic for those orders taking into account the values of $\theta_{1}^{\infty}$ and $\theta_{2}^{\infty}$ from Table 1 , see [7, p. 1184]. 
The effect of rounding errors on the evaluation of the matrix polynomial $h_{m}(\lambda, A)$ can be bounded analogously to the numerator of Padé approximants in [7, p. 1185]. Using Theorem 2.2 of [7, p. 1184], taking into account that $\|A\|_{1} \leq \theta_{m}, e^{-\|A\|} \leq\left\|e^{A}\right\|$, and noting that $h_{m}(\lambda, A)$ has all positive coefficients for $\lambda \geq 1$, it follows that

$$
\begin{aligned}
\left\|h_{m}(\lambda, A)-\hat{h}_{m}(\lambda, A)\right\|_{1} & \leq \widetilde{\gamma}_{m n} h_{m}\left(\lambda,\|A\|_{1}\right) \approx \widetilde{\gamma}_{m n} e^{\|A\|_{1}} \leq \widetilde{\gamma}_{m n}\left\|e^{A}\right\|_{1} e^{2\|A\|_{1}} \\
& \simeq \widetilde{\gamma}_{m n}\left\|h_{m}(\lambda, A)\right\|_{1} e^{2\|A\|_{1}} \leq \widetilde{\gamma}_{m n}\left\|h_{m}(\lambda, A)\right\|_{1} e^{2 \theta_{m}},(26)
\end{aligned}
$$

where $A \in \mathbb{C}^{n \times n}, \hat{h}_{m}(\lambda, A)$ is the computed Hermite approximation using explicit formation of matrix powers as in $(23)$, and $\widetilde{\gamma}_{k}=c k u /(1-c k u)$ with $c$ a small integer constant [15]. Hence, the relative error is bounded approximately by $\widetilde{\gamma}_{m n} e^{2 \theta_{m}}$, which is a satisfactory bound taking into account the values of $\theta_{m}$ given in Tables 1 and 2. Analogously, a similar bound can be obtained for Taylor matrix polynomial.

We have implemented two algorithms for computing the matrix exponential by the Hermite method presented in this paper. The first algorithm (dgeexfher) computes, for double precision general matrices, the exponential function by a Hermite approximation using the classical Horner's and PatersonStockmeyer methods [13], [16, p. 568], [14, p. 73].

The second algorithm saves computational cost taking into account the relative accuracy bounds in IEEE double precision arithmetic. The underlying idea is that if the contribution of the highest degree terms of the Hermites series to the exponential of the scaled matrix is negligible taking into account floating point arithmetic bounds, then we can save the evaluation of matrix products without substantial changes in the final result. For example, for $m=9$, let

$$
F=p_{9} A^{3}+p_{8} A^{2}+p_{7} A,
$$

where $p_{i}, i=0,1, \cdots, 9$, are the coefficients of the matrix powers $A^{i}$ of the corresponding Hermite expansion in Table 3. Since

$$
\frac{\left\|p_{9} A^{9}+p_{8} A^{8}+p_{7} A^{7}\right\|}{\left\|e^{A}\right\|} \leq \frac{\|F\|\left\|A^{3}\right\|^{2}}{e^{-\|A\|}}
$$

if

$$
\frac{\min \left\{\|F\|,\left\|F+p_{6} I\right\|\right\}\left\|A^{3}\right\|^{2}}{e^{-\|A\|}}<u
$$


or

$$
\|F\|<\left|p_{6}\right| u,
$$

then matrix $F$ or matrix $F+p_{6} I$ can be neglected saving one matrix product. This is likely to occur for instance if the norm of matrix $A$ was only slightly greater than $\theta_{6}$. Similar tests can be devised and applied recursively, eliminating sets of 3 terms each time. It is important to note that if the condition is accomplished more than once or twice in the evaluation of $h_{m}(\lambda, A)$ for a scaled matrix $A$, it might be a sign of overscaling.

With respect to the computational cost of the bound test, if we denote $B$ the original unscaled matrix, then $A=B / 2^{s}$. Therefore, when doing the tests, the norm of the original unscaled matrix and the matrix power $A^{3}$ are already obtained. Hence, making the recursive tests only involves in practice to evaluate once at the beginning the norm of $A^{3}$, the expression $u e^{-\|A\|_{1}}=u e^{-\|B\|_{1} / 2^{s}}$, and two matrix norms for each test. The cost of these operations for $r \times r$ matrices is of order $O\left(r^{2}\right)$, negligible when compared to a matrix product, whose cost is of order $O\left(r^{3}\right)$.

Similar tests can be applied to the evaluation of Taylor approximation of orders $m=4,6,9,16,20$, and $h_{m}(\lambda, A)$ with $m=25,30$, giving Algorithm 2 (dgeexfhrp) which computes, for double precision general matrices, the exponential function by scaling-squaring Hermites approximation reducing the number of matrix products when possible. This algorithm has essentially the same stages as Algorithm 1, checking the tests when needed and potentially saving matrix products. Numerical tests will show that in practically the $100 \%$ of the test matrices the savings are obtained with the same accuracy in double precision arithmetic. Both algorithms 1 and 2 do not consider orders $m=1$ and 2 because $\|A\|$ should be very small to use those orders, given the low values of $\theta_{1}^{\infty}$ and $\theta_{2}^{\infty}$, see Table 1 .

Algorithm 1 can be divided into the following stages (algorithm 2 can be divided in analogous stages):

1. Preprocessing of matrix $A$ using the techniques proposed by Ward in [17] (steps 1-4). Note that in numerical tests we did not use preprocessing because turning it on provided similar comparison results to those turning it off.

2. After preprocessing, the optimal value of the scaling parameter $s$ is calculated (steps 5-20).

3. In steps 17 and 35-37 the matrix scaling and the squaring of the approximation is done, respectively. 
4. Finally in step 38 the postprocessing is applied. In the same way as preprocessing, this step has not been applied in numerical tests.

We have made available online a MATLAB sequential version of the complete algorithm dgeexfhrp at

$$
\text { http://personales.upv.es/ jorsasma/dgeexfhrp.zip, }
$$

which implements the rest of cases $m=9,12, \ldots, 30$ from lines $31-34$ of the algorithm, and offers the possibility to select the maximum order $m$.

\section{Numerical examples}

The main objective of this section is to compare MATLAB implementations of the algorithms developed in Section 3 with other efficient algorithms implemented in MATLAB that compute matrix exponential. MATLAB 7.7 (R2008b) implementations were tested on an Intel Core 2 Duo processor at $2.52 \mathrm{GHz}$ with $4 \mathrm{~GB}$ main memory. In the comparative the following MATLAB functions were used:

- Expm is a MATLAB function that uses Padé approximants of exponential function with scaling and squaring proposed by Higham in [7].

- Funm is a built-in MATLAB 7.7 function that enables computation of general matrix functions at square matrices. The matrix function must have a Taylor series with an infinite radius of convergence, except for the matrix logarithm, which is treated as a special case. The exponential, cosine, sine, hyperbolic sine, hyperbolic cosine and the logarithm of a matrix are all allowed. This function implements the Schur-Parlett algorithm of Davies and Higham [18].

Algorithm accuracy was tested by computing the relative error

$$
\mathrm{E}=\frac{\left\|e^{A}-\tilde{Y}\right\|_{1}}{\left\|e^{A}\right\|_{1}}
$$

where $\tilde{Y}$ is the computed solution and $e^{A}$ the exact solution.

As it is mentioned above, in the tests we did not use any preprocessing/postprocessing in the Hermite implemented algorithms. Analogously to the experiments in [7], we found that turning on preprocessing in this algorithm 

$\overline{\text { Algorithm } 1 \text { computes the exponential of a matrix by Hermite series with }}$
scaling and squaring and maximum order $m=30$.

Function $F=$ dgeexfher $(A)$

Input: Matrix $A \in \mathbb{C}^{r \times r} \quad$ Output: Matrix $F \cong e^{A} \in \mathbb{C}^{r \times r}$

1: $\mu=\operatorname{trace}(A) / r$

2: $A \leftarrow A-\mu I$

3: Determine a diagonal matrix $D$ and a permutation matrix $P$ such that $D^{-1} P^{T} A P D$ is balanced

4: $A \leftarrow D^{-1} P^{T} A P D \quad \triangleright$ Preprocessing of $A$

5: Initialize $\theta$ with values of Tables 5 , and coefficients $p_{i}$ for each approximation order $m=4,6,9, \cdots, 30$.

6: $\operatorname{norm} A=\|A\|_{1}$

7: for $m=\left[\begin{array}{llllll}4 & 9 & 12 & 16 & 202530\end{array}\right]$ do

8: $\quad$ if $\operatorname{norm} A \leq \theta_{m}$ then

9: $\quad$ break

10: end if

11: end for

12: if $\operatorname{norm} A>\theta_{30}$ then

13: $s=\left\lceil\log _{2}\left(\right.\right.$ norm $\left.\left.A / \theta_{30}\right)\right\rceil$

14: $\quad$ if $n o r m A / 2^{s} \leq \theta_{25}$ then $\quad \triangleright$ This condition can occur because $\theta_{30} / 2<\theta_{25}$

15: $\quad m=25$

16: end if

17: $\quad A \leftarrow A / 2^{s}$

18: else

19: $s=0$

20: end if

21: $A_{2}=A^{2}$

22: if $m==4$ then $\quad \triangleright$ Taylor approximation for $m=4,6,9,12,16,20$

23: $\quad F=\left(p_{4} A_{2}+p_{3} A+p_{2} I\right) A_{2}+p_{1} A+p_{0} I$

24: else if $m==6$ then

25: $\quad F=\left(\left(p_{6} A_{2}+p_{5} A+p_{4} I\right) A_{2}+p_{3} A+p_{2} I\right) A_{2}+p_{1} A+p_{0} I$

26: else if $m==9$ then

27: $\quad A_{3}=A \cdot A_{2}$

28: $\quad F=\left(\left(p_{9} A_{3}+p_{8} A_{2}+p_{7} A+p_{6} I\right) A_{3}+p_{5} A_{2}+p_{4} A+p_{3} I\right) A_{3}+p_{2} A_{2}+p_{1} A+p_{0} I$

29: else if $m==12$ then

30:

31:

33:

34: end if

35: for $k=1: s$ do

$\triangleright$ Squaring Phase: Repeated squaring of matrix $F$

36: $\quad F=F^{2}$

37: end for

38: $F \leftarrow e^{\mu} P D F D^{-1} P^{T}$

$\triangleright$ Postprocessing of $F$ 
Algorithm 2 computes the exponential of a matrix by scaling and squaring Hermite approach with maximum order $m=30$ reducing the number of matrix products when possible.

Function $F=\operatorname{dgeexfhrp}(A)$

Input: Matrix $A \in \mathbb{C}^{r \times r} \quad$ Output: Matrix $F \cong e^{A} \in \mathbb{C}^{r \times r}$

1: Same as dgeexfher(A) lines 1-21

2: $u=2^{-53}$

3: LowBound $=u e^{- \text {norm } A / 2^{s}}$

4: if $m==4$ then

5: $\quad F=p_{4} A_{2}+p_{3} A$

6: $\quad$ aux $=\|F\|_{1}$

7: $\quad F=F+p_{2} I$

8: $\quad$ if $\left(\right.$ aux $\left.\leq\left|p_{2}\right| u\right)$ or $\left(\min \left\{\right.\right.$ aux,$\left.\|F\|_{1}\right\} \cdot\left\|A_{2}\right\|_{1} \leq$ LowBound $)$ then

9: $\quad F=p_{2} A_{2}+p_{1} A+p_{0} I \quad \triangleright$ One matrix product is saved 10: $\quad$ else

11: $\quad F=F \cdot A_{2}+p_{1} A+p_{0} I$

12: $\quad$ end if

13: else if $m==6$ then

14: $\quad$ norm $A_{2}=\left\|A_{2}\right\|_{1}$

15: $\quad F=p_{6} A_{2}+p_{5} A$

16: $\quad$ aux $=\|F\|_{1}$

17: $\quad F=F+p_{4} I$

18: $\quad$ if $\left(\right.$ aux $\left.\leq\left|p_{4}\right| u\right)$ or $\left(\min \left\{\right.\right.$ aux,$\left.\|F\|_{1}\right\} \cdot\left\|A_{2}\right\|_{1}^{2} \leq$ LowBound $)$ then

19: $\quad F=p_{4} A_{2}+p_{3} A \quad \triangleright$ One matrix product is saved 20: else

21: $\quad F=F \cdot A_{2}+p_{3} A$

22: end if

23: $\quad$ aux $=\|F\|_{1}$

24: $\quad F=F+p_{2} I$

25: $\quad$ if $\left(\right.$ aux $\left.\leq\left|p_{2}\right| u\right)$ or $\left(\min \left\{a u x,\|F\|_{1}\right\} \cdot\left\|A_{2}\right\|_{1} \leq\right.$ LowBound $)$ then

26: $\quad F=p_{2} A_{2}+p_{1} A+p_{0} I \quad \triangleright$ One matrix product is saved 27: else

28: $\quad F=F \cdot A_{2}+p_{1} A+p_{0} I$

29: $\quad$ end if

30: else if $m==9$ then

31:

32:

33: else if $m==30$ then

34:

35: end if

36: Same as dgeexfher(A) lines 35-38 
provided similar results to those presented in this section without preprocessing.

Regarding memory issues, it is important to note that Algorithm 1 needs the same matrices in memory as expm when both methods use their maximum orders, $m=30$ and $m=13$ respectively: $A, A^{2}, \ldots, A^{5}$ plus one to perform the calculation for Hermite method, and $A, A^{2}, A^{4}, A^{6}$ plus two for the numerator and denominator for Padé method, taking into account that the final rational approximation can be performed re-using the memory allocated for the power of $A$ involved in the numerator and denominator computation.

Regarding computational cost, from Table 4 and Table 2.3 of [7], Table 5 presents the orders of the approximation, the $\theta_{m}$ and $\theta_{m}^{\prime}$ values and the number of matrix products $\pi_{m}$ and $\pi_{m}^{\prime}$ required for Hermite dgeexfher function, and Padé expm function, respectively. Note that the number of matrix products for dgeexfher is a maximum bound of the matrix products for dgeexfhrp. Then, using (24), for matrices with $\|A\|>\theta_{13}^{\prime}=5.37$ (showing three significant digits), the cost of dgeexfher in terms of matrix products, denoted by $C_{m}^{H}$, and representing the maximum cost of dgeexfhrp, is

$$
\begin{aligned}
& C_{30}^{H}=9+s_{H}=9+\left\lceil\log _{2}\|A\|-\log _{2}(3.58)\right\rceil, \text { if } \frac{\|A\|}{2^{s_{H}}}>2.44, \\
& C_{25}^{H}=8+s_{H}=8+\left\lceil\log _{2}\|A\|-\log _{2}(3.58)\right\rceil, \text { if } \frac{\|A\|}{2^{s_{H}}} \leq 2.44,
\end{aligned}
$$

where $s_{H}$ denotes the scaling in Hermite methods. On the other hand, the cost of expm Padé method, denoted by $C_{m}^{P}$, is

$$
C_{13}^{P}=6+C_{L S}+s_{P}=6+C_{L S}+\left\lceil\log _{2}\|A\|-\log _{2}(5.37)\right\rceil,
$$

where

$$
s_{P}=\left\lceil\log _{2}\|A\|-\log _{2}(5.37)\right\rceil,
$$

denotes the scaling in expm and $C_{L S}$ denotes the cost of solving the multiple right-hand sides linear system in Padé method, in terms of matrix products. From [19] the cost of the matrix product in $\mathbb{R}^{r \times r}$ and the solution of the multiple right-hand sides of the same size with Padé approximants is $2 r^{3}-r^{2}$ and $\frac{8 r^{3}}{3}-\frac{r^{2}}{2}+\frac{5 r}{6}$ flops, respectively. Therefore, asymptotically $C_{L S} \approx 4 / 3$.

Taking into account that $\theta_{30}$ for Hermite methods is greater than $\theta_{13}^{\prime} / 2$ for Padé method [7, p. 1186], taking expm's matrix scaling by $2^{s_{P}}$, for matrices with

$$
\theta_{13}^{\prime} / 2=2.68<\frac{\|A\|}{2^{s_{P}}} \leq 3.58
$$


Hermite methods use $m=30$ and $s_{H}=s_{P}$, therefore dgeexfhrp needs a maximum of $3-C_{L S}$ more matrix products than expm, which results in a maximum relative higher cost of $(1+2 / 3) /\left(7+1 / 3+s_{P}\right) \times 100 \% \leq 20 \%$ in matrix products, which decreases with the matrix norm because of the increasing scaling. For matrices such that

$$
3.58<\frac{\|A\|}{2^{s_{P}}} \leq 2 \times \theta_{25}=4.88
$$

Hermite methods use $m=25$ and $s_{H}=s_{P}+1$, therefore dgeexfhrp needs a maximum of $3-C_{L S}$ more matrix products than expm again, resulting in the same relative higher cost $(1+2 / 3) /\left(7+1 / 3+s_{P}\right) \times 100 \% \leq 20 \%$ in matrix products, decreasing with the matrix norm. In fact, using (35) and (36), for instance for matrices with norm

$$
343.80<\|A\| \leq 624.98
$$

it follows that $s_{P}=7$ and the maximum relative higher cost of dgeexfhrp decreases to $11.63 \%$. Finally, for matrices such that

$$
4.88<\frac{\|A\|}{2^{s_{P}}} \leq 5.37
$$

Hermite methods use $m=30$ and $s_{H}=s_{P}+1$, therefore dgeexfhrp needs a maximum of $4-C_{L S}$ more matrix products than expm, resulting in a maximum relative higher cost of $(2+2 / 3) /\left(7+1 / 3+s_{P}\right) \times 100 \% \leq 32 \%$ in matrix products. Note that this norm interval represents only the $18.21 \%$ of the total interval considered in the three cases (35), (36) and (38).

The cost comparison for matrices with $\|A\| \leq 5.37$ is presented in Table 6 , where the $\theta_{m}$ values are presented with three significant digits, and $C_{m}^{H}$ represents a bound on the maximum cost of dgeexfhrp. Note that there are some cases where the maximum cost for dgeexfhrp is lower than the cost for expm, i.e. $2.53 e-1<\|A\| \leq 2.99 e-1,1.49 e-2<\|A\| \leq 8.95 e-2$ and $\|A\| \leq 9.06 e-3$, reaching relative efficiency gains from $6.25 \%$ up to $40 \%$. For matrices satisfying $4.88 \leq\|A\| \leq 5.37$ the maximum cost of dgeexfhrp is $36.36 \%$ higher and in the rest of cases dgeexfhrp maximum cost exceeds expm cost in $2 / 3$ or $1+2 / 3$ matrix products. Once again the interval where the cost difference is higher is a small part of all the interval considered, representing only the $9.11 \%$ of the total. One more final squaring step than in expm is required for matrices satisfying $3.58<\|A\| / 2^{s_{P}} \leq 5.37$ with $\|A\|>5.37$, and $3.58<\|A\| \leq 5.37$. This might be a possible source of error, especially if $A$ 


\begin{tabular}{c|ccc|ccc|c}
\hline & \multicolumn{3}{|c|}{ dgeexfhrp } & \multicolumn{3}{|c|}{ expm } & \\
\hline Norm ranges & $m$ & $s_{H}$ & $C_{m}^{H}$ & $m$ & $s_{P}$ & $C_{m}^{P}$ & $\frac{C_{m}^{H}-C_{m}^{P}}{C_{m}^{P}} \%$ \\
\hline$\|A\| \leq 3.39 e-4$ & 4 & 0 & 2 & 3 & 0 & $2+C_{L S}$ & -40 \\
$3.39 e-4<\|A\| \leq 9.06 e-3$ & 6 & 0 & 3 & 3 & 0 & $2+C_{L S}$ & -10 \\
$9.06 e-3<\|A\| \leq 1.49 e-2$ & 9 & 0 & 4 & 3 & 0 & $2+C_{L S}$ & 20 \\
$1.49 e-2<\|A\| \leq 8.95 e-2$ & 9 & 0 & 4 & 5 & 0 & $3+C_{L S}$ & -7.69 \\
$8.95 e-2<\|A\| \leq 2.53 e-1$ & 12 & 0 & 5 & 5 & 0 & $3+C_{L S}$ & 15.38 \\
$2.53 e-1<\|A\| \leq 2.99 e-1$ & 12 & 0 & 5 & 7 & 0 & $4+C_{L S}$ & -6.25 \\
$2.99 e-1<\|A\| \leq 7.80 e-1$ & 16 & 0 & 6 & 7 & 0 & $4+C_{L S}$ & 12.50 \\
$7.80 e-1<\|A\| \leq 9.50 e-1$ & 20 & 0 & 7 & 7 & 0 & $4+C_{L S}$ & 31.25 \\
$9.50 e-1<\|A\| \leq 1.43$ & 20 & 0 & 7 & 9 & 0 & $5+C_{L S}$ & 10.53 \\
$1.43<\|A\| \leq 2.09$ & 25 & 0 & 8 & 9 & 0 & $5+C_{L S}$ & 26.32 \\
$2.09<\|A\| \leq 2.44$ & 25 & 0 & 8 & 13 & 0 & $6+C_{L S}$ & 9.09 \\
$2.44<\|A\| \leq 3.58$ & 30 & 0 & 9 & 13 & 0 & $6+C_{L S}$ & 22.73 \\
$3.58<\|A\| \leq 2 \times 2.44$ & 25 & 1 & 9 & 13 & 0 & $6+C_{L S}$ & 22.73 \\
$2 \times 2.44<\|A\| \leq 5.37$ & 30 & 1 & 10 & 13 & 0 & $6+C_{L S}$ & 36.36 \\
\hline
\end{tabular}

Table 6: Comparison of maximum theoretical cost $C_{m}^{H}$ in terms of matrix products for dgeexfhrp with maximum order $m=30$, and cost $C_{m}^{P}$ for expm, for $\|A\| \leq 5.37$.

is ill-conditioned. However, Hermite methods with maximum order $m=30$ obtained better accuracy than expm in a high percentage of cases in numerical tests (see Table 8).

In a similar way, it is easy to show that, for any matrix $A \in \mathbb{C}^{r \times r}$, the maximum cost of using dgeexfhrp with maximum order $m=16$ is the same as using $m=20$. Thus, maximum order $m=20$ should be used instead of 16 because taking into account that $\theta_{20}>\theta_{16}$, the scaling parameter $s$ with $m=20$ is lower in the majority of matrix norm intervals, and the squaring process might be a possible source of error. Analogously, it is also easy to check that if we use maximum orders $m=20$ or 25 then dgeexfhrp presents a maximum higher cost than expm of $1+2 / 3$ matrix multiplications, instead of $2+2 / 3$ that dgeexfhrp presented with $m=30$. On the other hand, dgeexfhrp with maximum orders $m=20$ and 25 presents lower maximum cost than expm for the same matrix norms as dgeexfhrp with $m=30$.

It is important to note that all the costs of dgeexfhrp presented in this analysis are maximum costs and, as we will see in tests, they may decrease considerably in practice.

For tests, 105 matrices were used: 49 matrices from the Matrix Computation Toolbox [20], 24 matrices from the Eigtool MATLAB package [21], 18 
Table 7: Relative error comparison (\%) between dgeexfher and dgeexfhrp.

\begin{tabular}{ccccc}
\hline maximum order & $m=16$ & $m=20$ & $m=25$ & $m=30$ \\
\hline$E_{\text {dgeexfher }}<E_{\text {dgeexfhrp }}$ & 3.77 & 0 & 0 & 0 \\
$E_{\text {dgeexfher }}=E_{\text {dgeexfhrp }}$ & 95.28 & 99.06 & 100.00 & 100.00 \\
$E_{\text {dgeexfher }}>E_{\text {dgeexfhrp }}$ & 0.94 & 0.94 & 0.00 & 0.00 \\
$\mathrm{P}_{\text {dgeexfher }} / \mathrm{P}_{\text {dgeexfhrp }}$ & 105.52 & 108.43 & 108.58 & 109.91 \\
\hline
\end{tabular}

matrices from papers of the state-of-the-art of matrix functions $[6,17,18$, $22,23,24,25,26]$, and 14 special matrices such as matrices of Vandermonde, Hankel, Toeplitz, Wilkinson, symmetric matrices, defective matrices and non defective matrices.

In the examples the matrix exponentials were calculated analytically, when it was possible, or by using [33/33] diagonal Padé method with scaling and squaring with 1000-digit precision in an iterative way: different increasing scalings starting from that provided in [7] for expm were used, until the norm of the relative difference between the approximations converted to IEEE double precision arithmetic was zero in four iterations. The [33/33] diagonal Padé approximation was evaluated with matrix power aggregation similar to that proposed in [7, p. 1183].

Table 7 shows a comparative between the implementations dgeexfher and dgeexfhrp. The three first rows contain the percentage of times that relative error of a function is lower, equal or greater than relative error of the other function. The last row of Table 7 contains the ratio of the total number of matrix products evaluated for the two functions over all test matrices, denoted by $\mathrm{P}_{\text {dgeexfher }}$ and $\mathrm{P}_{\text {dgeexfhrp }}$. Both functions presented practically the same accuracy, however the total number of matrix products evaluated for dgeexfhrp is lower than those for dgeexfher.

Table 8 presents the relative error and matrix product comparison of dgeexfhrp and dgeexfher with expm. dgeexfher obtained the same comparative results of accuracy as dgeexfhrp at a higher cost, see the last two rows. dgeexfhrp relative error is lower than expm relative error for $m \geq 20$ (68.87\%-77.36\%), with a slightly higher cost, varying the ratio of matrix products between $103.76 \%$ and $104.43 \%$ for $m=20,25,30$.

In Table 9 the relative errors of dgeexfhrp and dgeexfher are compared to funm. As shown in these tables, once again dgeexfher and dgeexfhrp obtained the same comparative results of accuracy, and in general their relative 
Table 8: Comparison of relative error and total number of matrix products (\%) between dgeexfhrp and expm. The error comparison results were exactly the same with dgeexfher at a greater cost (see last table row).

\begin{tabular}{ccccc}
\hline maximum order & $m=16$ & $m=20$ & $m=25$ & $m=30$ \\
\hline $\mathrm{E}_{\text {dgeexfhrp }}<\mathrm{E}_{\text {expm }}$ & 44.34 & 68.87 & 77.36 & 77.36 \\
$\mathrm{E}_{\text {dgeexfhrp }}=\mathrm{E}_{\text {expm }}$ & 1.89 & 2.83 & 1.89 & 1.89 \\
$\mathrm{E}_{\text {dgeexfhrp }}>\mathrm{E}_{\text {expm }}$ & 53.77 & 28.30 & 20.75 & 20.75 \\
$\mathrm{P}_{\text {dgeexfhrp }} / \mathrm{P}_{\text {expm }}$ & 106.62 & 103.76 & 104.01 & 104.43 \\
$\mathrm{P}_{\text {dgeexfher }} / \mathrm{P}_{\text {expm }}$ & 112.51 & 112.51 & 112.93 & 114.78 \\
\hline
\end{tabular}

Table 9: Relative error comparison between dgeexfhrp and funm. The results were exactly the same with dgeexfher.

\begin{tabular}{ccccc}
\hline maximum order & $m=16$ & $m=20$ & $m=25$ & $m=30$ \\
\hline $\mathrm{E}_{\text {dgeexfhrp }}<\mathrm{E}_{\text {funm }}$ & 71.70 & 77.36 & 79.25 & 80.19 \\
$\mathrm{E}_{\text {dgeexfhrp }}=\mathrm{E}_{\text {funm }}$ & 0.94 & 0 & 0 & 0 \\
$\mathrm{E}_{\text {dgeexfhrp }}>\mathrm{E}_{\text {funm }}$ & 28.30 & 22.64 & 20.75 & 19.81 \\
\hline
\end{tabular}

errors were lower than the relative errors of funm $(71.70 \%-80.19 \%)$.

Figure 2a presents the normwise relative errors of dgeexfhrp, expm and funm (a similar figure is obtained when dgeexfher is used). This figure shows the relative error of all implementations sorted in decreasing order, and a solid line that represents the unit roundoff multiplied by the relative condition number of the exponential function at $X[14$, p. 56],

$$
\operatorname{cond}_{\exp }(X)=\lim _{\varepsilon \rightarrow 0} \sup _{\|E\| \leq \varepsilon} \frac{\left\|e^{X+E}-e^{X}\right\|}{\varepsilon} \frac{\|X\|}{\left\|e^{X}\right\|} .
$$

Relative condition number was computed using the MATLAB function expm_cond. This function is incorporated into the Matrix Function Toolbox developed by Higham [14, Appendix D] and available at http://www.ma.man.ac.uk/ higham/mftoolbox.

For a method to perform in a backward and forward stable manner, its error should lie not far above this line on the graph [7, p. 1188]. Figure 2a shows that all functions perform in a numerically stable way on this test, even for matrices 64-70 where there were overscaling problems [26]. 


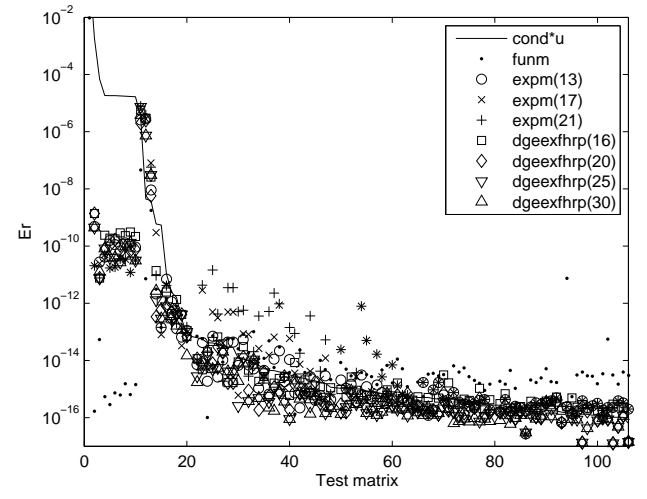

(a) Normwise relative errors

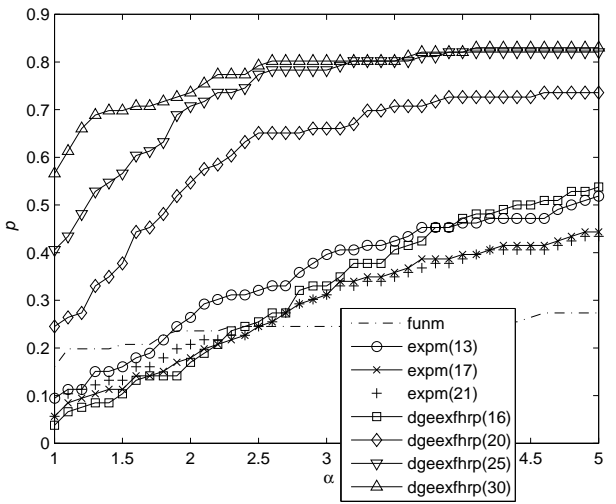

(b) Performance profile

Figure 2: Comparative of dgeexfhrp, expm and funm.

Performance profile [27] is presented in Figure 2b. This figure shows the performances of the compared functions, where $\alpha$ coordinate varies between 1 and 5 in steps equal to 0.1 , and $p$ coordinate is the probability that the considered function has a relative error lower or equal than $\alpha$-times the smallest error over all the methods. The probabilities are defined over all matrices considered in the tests. As shown in this figure, dgeexfhrp with maximum order $m=30$ is the most accurate function, and it was achieved with very similar cost to dgeexfhrp with maximum orders $m=16,20$ or 25 . Hence, we consider $m=30$ as the best choice of maximum order for dgeexfhrp.

\section{Conclusions}

In this work an efficient method to approximate the matrix exponential based on Hermite matrix polynomial expansions has been presented. Following the ideas of [7] we have developed an optimal backward error bound for the scaling and squaring Hermite method in exact arithmetic, which depends on $A$ only through $\|A\|$ and enables to obtain the theoretical optimal scaling for general matrices. The optimal parameter $\lambda$ of the algorithm has been obtained for each order $m=21,22, \ldots, 30$, providing greater values of the Hermite scaling parameter $\theta_{m}$ than Taylor methods for those orders, i.e. $\theta_{m}>\theta_{m}^{\infty}, m=21,22, \ldots, 30$, where $\theta_{m}^{\infty}$ is the scaling parameter for Taylor methods, see Tables 1 and 2. Based on that result, two mixed Hermite-Taylor algorithms have been developed in order to evaluate different order matrix 
polynomial approximations: a Hermite-Taylor series Paterson-Stockmeyer algorithm, dgeexfher, and a modified algorithm, dgeexfhrp, which taking into account floating point arithmetic error bounds may reduce the number of matrix product evaluations. This modification (dgeexfhrp) presented practically the same accuracy as dgeexfher in numerical tests with a lower computational cost of up to $9.91 \%$. Both algorithms use Hermite series for order $m>20$ and Taylor series for order $m \leq 20$. From experimental results, we have identified the best choice of maximum degree $m$ of Hermite approximation in terms of efficiency and accuracy: $m=30$.

We have shown that dgeexfhrp with maximum order $m=30$ has lower theoretical maximum cost than expm for some matrix norm intervals, and in numerical tests the cost of dgeexfhrp was similar to that for expm.

dgeexfhrp stores the same number of matrices in memory as expm when both functions use their maximum orders, $m=30$ and $m=13$ respectively, and does not need the solution of multiple linear systems. [7] shows that this solution does not introduce large errors in expm. However, dgeexfhrp obtained higher accuracy than both funm and expm in the majority of test matrices, i.e. the $80.19 \%$ and $77.36 \%$ respectively. These results are based on empirical observations. However, numerical results are promising and further research on Hermite matrix polynomial series for the matrix exponential is being carried out to reduce the computational costs.

\section{Acknowledgements}

This work has been supported by the Programa de Apoyo a la Investigación y el Desarrollo of the Universidad Politécnica de Valencia PAID-0509-4338, 2009.

\section{References}

[1] R. A. Frazer, W. J. Duncan, A. R. Collar, Elementary Matrix and Some Applications to Dynamics and Differential Equations, Macmillan, New York, 1946.

[2] M. W. Hirsch, S. Smale, Differential Equations, Dynamical Systems and Linear Algebra, Academic Press, New York, 1974.

[3] T. Kailath, Linear Systems, Prentice-Hall, Englewood Cliffs, NJ, 1980. 
[4] C. B. Moler, C. V. Loan, Nineteen dubious ways to compute the exponential of a matrix, SIAM Review 20 (4) (1978) 801-836.

[5] G. D. Smith, Numerical Solution of Partial Differential Equations: Finite Difference Methods, $3^{\text {rd }}$ Edition, Oxford University Press, 1985.

[6] C. B. Moler, C. V. Loan, Nineteen dubious ways to compute the exponential of a matrix, twenty-five years later, SIAM Rev. 45 (2003) 3-49.

[7] N. J. Higham, The scaling and squaring method for the matrix exponential revisited, SIAM J. Matrix Anal. Appl. 26 (4) (2005) 1179-1193.

[8] A. H. Al-Mohy, N. J. Higham, A new scaling and squaring algorithm for the matrix exponential, SIAM J. Matrix Anal. Appl. 31 (3) (2009) 970-989.

[9] N. Dunford, J. Schwartz, Linear Operators, Part I., Interscience Publishers, New York, 1957.

[10] S. Saks, A. Zygmund, Analytic Functions, Elsevier Science Publishers, Amsterdam, The Netherlands, 1971.

[11] E. Defez, L. Jódar, Some applications of Hermite matrix polynomials series expansions, J. Comput. Appl. Math. 99 (1998) 105-117.

[12] L. Jódar, R. Company, Hermite matrix polynomials and second order matrix differential equations, J. Approximation Theory Appl. 12 (2) (1996) 20-30.

[13] M. S. Paterson, L. J. Stockmeyer, On the number of nonscalar multiplications necessary to evaluate polynomials, SIAM J. Comput. 2 (1) (1973) 60-66.

[14] N. J. Higham, Functions of Matrices: Theory and Computation, Society for Industrial and Applied Mathematics, Philadelphia, PA, USA, 2008.

[15] N. J. Higham, Accuracy and Stability of Numerical Algorithms, 2nd Edition, Society for Industrial and Applied Mathematics, Philadelphia, PA, USA, 2002.

[16] G. H. Golub, C. V. Loan, Matrix Computations, 3rd Ed., Johns Hopkins Studies in Math. Sci., The Johns Hopkins University Press, 1996. 
[17] R. C. Ward, Numerical computation of the matrix exponential with accuracy estimate, SIAM J. Numer. Anal. 14 (4) (1977) 600-610.

[18] P. I. Davies, N. J. Higham, A Schur-Parlett algorithm for computing matrix functions, SIAM J. Matrix Anal. Appl. 25 (2) (2003) 464-485.

[19] S. Blackford, J. Dongarra, Installation guide for LAPACK, LAPACK Working Note 411, Department of Computer Science University of Tenessee (1999).

[20] N. J. Higham, The Test Matrix Toolbox for MATLAB, Numerical Analysis Report No. 237, Manchester Centre for Computational Mathematics, Manchester, England (Dec. 1993).

[21] T. G. Wright, Eigtool, version 2.1, (2009) http://web.comlab.ox.ac.uk/pseudospectra/eigtool/

[22] I. Najfeld, T. F. Havel, Derivatives of the matrix exponential and their computation, Advances in Appl. Math. 16 (1995) 321-375.

[23] C. S. Kenney, A. J. Laub, A Schur-Fréchet algorithm for computing the logarithm and exponential of a matrix, SIAM J. Matrix Anal. Appl. 19 (3) (1998) 640-663.

[24] D. Westreich, A practical method for computing the exponential of a matrix and its integral, Communications in Appl. Numer. Methods 6 (1990) 375-380.

[25] Y. Y. Lu, Computing a matrix function for exponential integrators, J. Comput. Appl. Math. 161 (2003) 203-216.

[26] L. Dieci, A. Papini, Padé approximation for the exponential of a block triangular matrix, Linear Algebra Appl. 308 (2000) 183-202.

[27] E. D. Dolan, J. J. Moré, Benchmarking optimization software with performance profiles, Math. Programming 91 (2002) 201-213. 